\title{
Ionic and Conformational Mobility in Poly(vinylidene fluoride)/Ionic Liquid blends: Dielectric and Electrical Conductivity Behavior
}

D. M. Correia ${ }^{1}$, R. Sabater i Serra ${ }^{2,3}$, J.A Gómez Tejedor ${ }^{2}$, V. de Zea Bermudez ${ }^{1}$ A. Andrio Balado4, J. M. Meseguer-Dueñas ${ }^{2,3}$, J. L. Gomez Ribelles ${ }^{2,3}$, S. LancerosMéndez ${ }^{5,6}$, C. M. Costa $^{7 *}$

${ }^{1}$ Departament of Chemistry and CQ-VR, University of Trás-os-Montes e Alto Douro, 5001-801Vila Real, Portugal

${ }^{2}$ Centre for Biomaterials and Tissue Engineering, CBIT, Universitat Politècnica de València, 46022 Valencia, Spain

${ }^{3}$ Biomedical Research Networking Center on Bioengineering, Biomaterials and Nanomedicine (CIBER-BBN), Valencia, Spain

${ }^{4}$ Departament de Física, Universitat Jaume I, 12071 Castelló, Spain ${ }^{5}$ BCMaterials, Basque Center for Materials, Applications and Nanostructures, UPV/EHU Science Park, 48940 Leioa, Spain

${ }^{6}$ IKERBASQUE, Basque Foundation for Science, 48013, Bilbao, Spain

${ }^{7}$ Centro de Física, Universidade do Minho, 4710-057 Braga, Portugal

\section{* Corresponding Author:}

C.M. Costa (․mscosta@fisica.uminho.pt)

Keywords: PVDF; ionic liquid; dielectric response; conductivity; electric modulus 
Abstract: The glass transition dynamics and the charge transport for blends composed of poly(vinylidene fluoride (PVDF) and the ionic liquid (IL) 1-ethyl-3-methylimidazolium bis(trifluoromethylsulfonyl) imide, [Emim][TFSI] have been investigated as a function of different IL content $(0,10,25$ and $40 \% \mathrm{wt})$ by differential scanning calorimetry (DSC), dynamic-mechanical analysis (DMA) and broadband dielectric relaxation spectroscopy (BDS) in wide frequency and temperature ranges $\left(0.1 \mathrm{~Hz}\right.$ to $1 \mathrm{MHz}$ and -120 to $150{ }^{\circ} \mathrm{C}$, respectively). The inclusion of the IL in the polymer matrix affected the main relaxation process ( $\beta$-relaxation) of the amorphous phase of the polymer matrix detected with all the techniques employed. It is demonstrated, that the chain segments of PVDF and the IL are mixed at the nanometer range. The blends were homogeneous regardless of the amount of IL and the glass transition temperature $(\mathrm{Tg})$ shifted to lower temperatures as the IL content was increased. A good agreement between the Tg measured by BDS and DSC was observed for all PVDF/IL samples. The conductivity formalism revealed significant contributions of the IL concentration to the conductivity behavior of the blends in that is described by charge motion and electrode polarization effects. The activation energy of all the PVDF/IL samples, calculated by Dyre model, decreased with IL addition with respect to that of neat PVDF. 


\section{Introduction}

In recent years, advances in technology for portable devices, but also for transport applications, such as hybrid electric vehicles (PHEV) and electric vehicles (EV), has led to an increasing demand in the battery sector for improvement of the batteries' safety, energy density and power, and also for the reduction of their cost and weight [1].

The transport sector requires an improvement of the energy density of lithium (Li) ion batteries of 2 to 5 times, while reducing cost and considering environmental (safety) problems $[2,3]$. The polymer electrolyte $(\mathrm{PE})$ in the lithium ion battery devices can play an important role to achieve these goals [4].

PEs are a (ionic salts and/or ionic liquids (ILs)) a class of macromolecular systems with a high ionic conductivity $\left(>10^{-7}{\mathrm{~S} . \mathrm{cm}^{-1}}^{-1}[5,6]\right.$. Basically, these materials are composed of guest species (ionic salts or ionic liquids (ILs)) dissolved in a host polymeric matrix. Different nanofillers (ceramic, metallic, etc.) can be added mainly to further improve the mechanical and electrochemical properties [7-9].

In this research field, it has been recognized that poly(vinylidene fluoride) (PVDF) is a polymeric matrix of significant interest because of its strong polarity (high dipolar moment) and high dielectric constant with respect to other polymeric materials, which can aid the ionization of ionic salts [10]. In addition, PVDF is known for its electroactive behavior (piezo, pyro and ferroelectric properties) and its good chemical and thermal stability, as well as excellent mechanical properties $[11,12]$. The piezoelectric properties of PVDF are particularly attractive for the application in Li ion batteries. It has been shown that in a conventional lithium ion battery, the piezoelectric potential of a PVDF film, created by mechanical stress, acts as a charge pump to drive $\mathrm{Li}^{+}$ions to migrate from the cathode to the anode following the reactions of load on the electrodes improving its performance $[13,14]$. 
ILs are one the more propitious compounds for Li ion batteries, since they solve safety problems due of their physicochemical features, including their good chemical and electrochemical stability, non-flammability, negligible vapor pressure and high ionic conductivity $[15,16]$.

ILs can be classed as aprotic or protic depending on the nature of cation, their structure and properties being largely determined by the choice of the cation and anion $[15,17]$. There are thousands of possible ILs available with a wide variety of properties, such as density, vapor pressure, viscosity, conductivity, diffusion, thermal stability, compatibilities and solubility $[18,19]$.

Various IL, such as 1-vinyl-3-butylimidazolium chloride [Vbim][Cl] [20, 21], 1-vinyl3-ethylimidazolium tetrafluoroborate $\quad\left([\mathrm{Veim}]\left[\mathrm{BF}_{4}\right]\right) \quad$ [22-24],1-butyl-3-

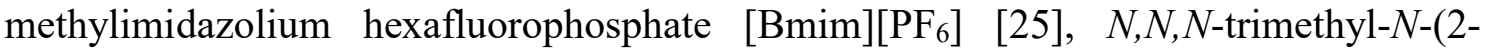
hydroxyethyl)ammonium bis(trifluoromethylsulfonyl)imide ([N $\mathrm{N}_{1} 11$ ethyl-3-methylimidazolium ethylsulfate ([Emim] $\left.]\left[\mathrm{C}_{2} \mathrm{SO}_{4}\right]\right)$ [26] and 1-ethyl-3methylimidazolium bis(trifluoromethylsulfonyl)imide ([Emim][TFSI]) [27] have been incorporated into the PVDF matrix in order to understand the interaction between the polymer chain and the IL, but also to improve PVDF's electrical, mechanical and thermal properties for electromechanical actuators. In this work, it was also demonstrated that, actuator deformation increases with increasing ionic liquid content, independently of the applied voltage [27].

In PVDF/IL composites, the ions may be present either as free ions, contact ion pairs or alternatively as diffusion ion pairs in which the IL are good glass-formers.

Understanding the ionic and molecular interactions between the ions (charged species) and the polymeric matrix, the formation of ion pairs, larger ion clusters, the contribution of ions to the electric properties (translation diffusion) and the dynamic glass transition 
are major issues for improving ionic conductivity in PEs [28, 29]. The ionic transport behavior depends of salt dissociation, salt concentration, dielectric constant of the host polymeric matrix, degree of ion aggregation and segmental mobility of the polymer chains [30].

Broadband dielectric spectroscopy (BDS) is widely used to obtain information about electric properties and molecular dynamics which can be related to the motion of the charged species and of the permanent dipoles in polymers [31, 32]. Thus, for instance, in the case of the poly(1-ethyl-3-vinylimidazolium bis(trifluoromethanesulfonylimide)) polymerized IL, BDS revealed the presence of three relaxation modes including that due to the effect of electrode polarization behavior [33]. The electrode polarization is an phenomenon that occurs in the interface between a metal surface related to the mechanism of charge transport at the interface [34]. In poly(ethylene oxide) (PEO)-based polyurethane ionomers containing mobile $\mathrm{Li}^{+}$or $\mathrm{Na}^{+}$ions BDS revealed the contributions of free ion concentration and ion mobility to electrode polarization [35].

Recently, the dielectric relaxation and conductivity of PEs composed of a small amount (0.01-10 wt \%) of the 1-ethyl-3-methylimidazolium nitrate ([Emim][ $\left.\left.\mathrm{NO}_{3}\right]\right)$ IL and PVDF were investigated by BDS. The dielectric response was found to be governed by a pronounced low-frequency relaxation which showed typical signatures of electrode polarization, whereas the main dipolar dielectric relaxation, associated with the glass transition, disappeared for IL contents higher than $1 \mathrm{wt} \%$, indicating that the amorphous phase lost its glass-forming properties and underwent structural changes [36]. On the other hand, DC conductivity and conductivity relaxation in PVDF/[BMIM][PF6] composites demonstrated that the incorporation of ILs into the matrix accelerated DC conductivity [37]. 
The goal of the present work is to analyze the miscibility, interaction between IL and the polymer chains of PVDF, and charge transport, through the analysis of the segmental mobility at the glass transition region of the polymer by Differential Scanning Calorimetry (DSC). The dielectric and dynamic-mechanical relaxation spectra were evaluated and the ionic mobility characterized by BDS. The IL used in this work was [Emim][TFSI] as it is a promising IL for battery applications owing to its high ionic conductivity and high thermal stability $[38,39]$. PVDF/IL blend films were prepared by solvent casting with high IL content (10-40 wt\%). 


\section{Experimental}

\subsection{Materials}

PVDF (Solef 6020, MW = $700 \mathrm{kDa}$ ), N,N-dimethylformamide (DMF, $99.5 \%$ ) and [Emim][TFSI] were acquired from Solvay, Merck and Iolitec, respectively.

\subsection{Preparation of the films}

PVDF was dissolved in DMF in a 15/85 weight ratio under magnetic stirring at room temperature. After the complete dissolution of the polymer, different contents of [Emim][TFSI] were added to the PVDF solution: 0, 10, 25 and $40 \%$ wt. $\mathrm{PVDF} /[$ Emim] $]$ TFSI] films $(\approx 50 \mu \mathrm{m}$ thick) were obtained after spreading the solution at room temperature on a glass substrate followed by solvent evaporation at $210{ }^{\circ} \mathrm{C}$ in an air oven. Thus, the blends will be identified henceforth as PVDF/[Emim][TFSI]X, where X is the IL content $(0,10,25$ and $40 \%)$.

\subsection{Characterization techniques}

Differential Scanning Calorimetry (DSC) was carried out with a DSC 8000 of PerkinElmer under a flowing nitrogen $\left(\mathrm{N}_{2}\right)$ atmosphere between $-120^{\circ} \mathrm{C}$ and $200{ }^{\circ} \mathrm{C}$ at a heating rate of $20^{\circ} \mathrm{C} / \mathrm{min}$. The mass of the sample was between 5 and $10 \mathrm{mg}$.

Dynamic-Mechanical Analysis (DMA) was performed in a PerkinElmer DMA 800 apparatus in tensile mode. The storage modulus and loss tangent were measured as a function of temperature at a frequency of $1 \mathrm{~Hz}$ from -90 to $90{ }^{\circ} \mathrm{C}$ at a heating rate of 2 ${ }^{\circ} \mathrm{C} / \mathrm{min}$. The typical dimension of each sample was $10 \times 4 \times 0.050 \mathrm{~mm}^{3}$.

Dielectric measurements were carried out in an impedance analyzer Alpha-S in a frequency range from $0.1 \mathrm{~Hz}$ to $1 \mathrm{MHz}$ where the temperature control was assured by a Quatro Cryosystem from Novocontrol GmbH. The sample cell with active head dielectric converter was mounted on a cryostat (BDS 1100) and exposed to a heated gas stream 
evaporated from a liquid $\mathrm{N}_{2}$ Deward. Circular gold electrodes (10 mm diameter) were deposited onto both sides of each sample, to form a parallel plate capacitor, by sputtering with a Polaron Coater SC502 under an argon atmosphere. The isothermal experiments were performed from -120 to $150{ }^{\circ} \mathrm{C}$ (thermal stability: $0.5^{\circ} \mathrm{C}$ ) in $5^{\circ} \mathrm{C}$ steps through the measurement of the capacitance (C) and the loss factor $(\tan \delta$ ), while the complex dielectric permittivity $\varepsilon^{*}=\varepsilon^{\prime}-\mathrm{i} \varepsilon^{\prime \prime}$ was determined as a function of frequency. 


\section{Results and discussion}

\subsection{Thermal and mechanical analysis}

The dispersion of IL into PVDF was investigated through SEM (see supplementary information of figure S1). The main microstructure of PVDF is maintained for the polymer blends with IL. On the other hand, it is observed a decrease of the average size of the spherulites, with addition of IL into the PVDF polymer. This behavior is due to the fact that the IL acts as nucleation centers for polymer crystallization [26, 27]. Further, the fact that the spherulitic growth is homogeneous along the sample indicates the good dispersion of the fillers.

The thermal behavior of the PVDF/[Emim][TFSI] samples was investigated by DSC. The thermograms recorded on heating scans are shown in Figure 1. PVDF/[Emim][TFSI] are blends of two glass-formers. Typically, the PVDF glass transition value reported in the literature is about $-40{ }^{\circ} \mathrm{C}[40]$ which is similar to that observed for pristine PVDF (Figure 1a).

As shown in the inset of Figure 1a, the thermogram of [Emim][TFSI] exhibits the glass transition in the temperature interval between -100 and $-88^{\circ} \mathrm{C}$ with a value of glass transition temperature $\left(\mathrm{T}_{\mathrm{g}}\right)$ of $-95{ }^{\circ} \mathrm{C}$. The exothermic peak around $-60{ }^{\circ} \mathrm{C}$ appears immediately after the glass transition and is ascribed to a cold crystallization. The endothermic peak emerging at higher temperatures with a maximum at $-2{ }^{\circ} \mathrm{C}$ corresponds to the melting temperature [41]. In both transitions, the existence of the double peak is due to the existence of various crystalline modifications of [Emim][TFSI] [41].

The thermogram of the PVDF/[Emim][TFSI] $40 \%$ blend is that expected for a miscible blend. It shows a broad glass transition with onset in the range of the glass transition of [Emim][TFSI] and ending around $-30^{\circ} \mathrm{C}$ as in PVDF (Figure 1a). The broad temperature 
of the glass transition is due to local fluctuations of the blend composition yielding local $\mathrm{T}_{\mathrm{g}}$ fluctuations.
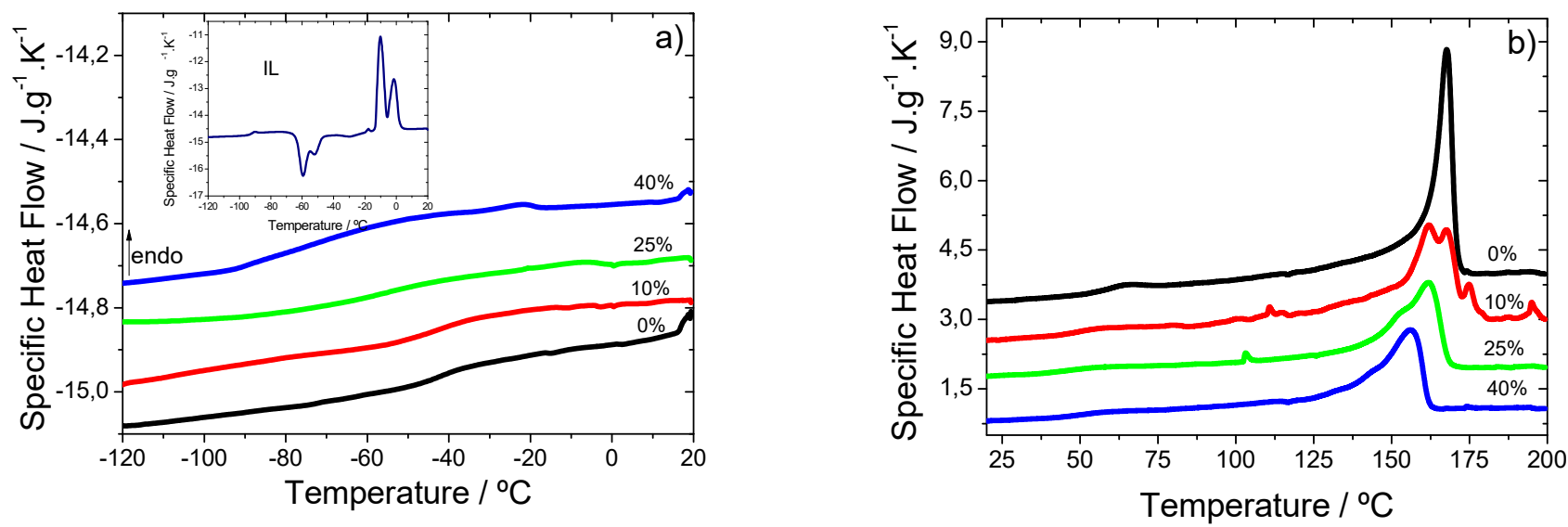

Figure 1. Specific heat flow per gram vs temperature for all PVDF/[Emim][TFSI] samples between -120 and $20^{\circ} \mathrm{C}(\mathrm{a})$, and between 25 and $200^{\circ} \mathrm{C}(\mathrm{b})$.

For intermediate compositions, the behavior is also similar to that of miscible blends, with the glass transition shifting towards lower temperatures as the [Emim][TFSI] content increases. The $T_{g}$ values era listed in Table 1. However, it should be noted that the blend $\mathrm{PVDF} /[\mathrm{Emim}][\mathrm{TFSI}] 10 \%$ has a $\mathrm{T}_{\mathrm{g}}$ practically equal to that of pure PVDF and this fact will be confirmed below, when analyzing the main mechanical or dielectric relaxation. In addition, it may be inferred from the Figure 1a) that for samples containing 25 and $40 \%$ of $[\mathrm{Emim}][\mathrm{TFSI}]$ the appearance of a small endothermic peak is seen at -25 and 10 ${ }^{\circ} \mathrm{C}$, respectively, that can may be correlated with the melting of small amounts of [Emim][TFSI] crystals.

Figure $1 \mathrm{~b}$ ) reproduces the DSC traces of the PVDF/[Emim][TFSI] samples between 25 and $200{ }^{\circ} \mathrm{C}$ showing the effect of the addition of the [Emim][TFSI] on the melting of PVDF. The values of the melting temperature $\left(T_{m}\right)$ and the degree of crystallinity $\left(\Delta \chi_{c}\right)$ are collected in Table 1 . The $\Delta \chi_{\mathrm{c}}$ value was calculated by the following equation [20]: 


$$
\Delta \chi_{C}=\frac{\Delta H_{m}}{\left(1-W_{I L}\right) \times \Delta H_{m}^{0}} \times 100
$$

where $\Delta H_{m}$ is the melting enthalpy of the PVDF/[Emim][TFSI] samples, $W_{I L}$ is the [Emim][TFSI] weight fraction in the PVDF/[Emim][TFSI] samples and $\Delta H_{m}^{0}$ is the melting enthalpy of fully crystalline PVDF (103.4 J/g) [42].

Table 1. Glass transition temperature $\left(\mathrm{T}_{\mathrm{g}}\right)$, melting temperature $\left(\mathrm{T}_{\mathrm{m}}\right)$ and degree of crystallinity $(\Delta \chi)$ for PVDF/[Emim][TFSI]X samples.

\begin{tabular}{|c|c|c|c|}
\hline Samples & $\mathbf{T}_{\mathbf{g}} /{ }^{\mathbf{O}} \mathbf{C}$ & $\mathbf{T}_{\mathbf{m}} /{ }^{\mathbf{0}} \mathbf{C}$ & $\mathbf{\Delta X} / \mathbf{\%}$ \\
\hline $\mathbf{0 \%}$ & -43 & 168 & 50 \\
\hline $\mathbf{1 0 \%}$ & -45 & 162 & 45 \\
\hline $\mathbf{2 5 \%}$ & -65 & 162 & 37.5 \\
\hline $\mathbf{4 0 \%}$ & -73 & 156 & 32.4 \\
\hline$[$ Emim] $[$ TFSI $]$ & -95 & ----- & ------- \\
\hline
\end{tabular}

Figure 1b) shows that the melting temperature $\left(T_{m}\right)$ and the corresponding melting area decreases with increasing IL content, where is verified a shift of the melting temperature to lower values with increasing [Emim][TFSI] content. The decrease of the Tm observed for the blend samples indicates a high miscibility between IL and PVDF [21].

Table 1 shows that $\mathrm{T}_{\mathrm{m}}$ and $\Delta \chi$ decreased with the increase of the [Emim][TFSI] amount. It can be deduced from these results that the IL destabilized the crystalline phase of the PVDF polymer due to the electrostatic interactions established between both components [43].

It has been reported in the literature that in the case of polymer blends with high IL content, there are two competitive effects on the crystallization behavior of the host polymer: on the one hand the promotion of polymer nucleation, but on the other, confinement effects leading to imperfections in the crystalline lamellae [44]. 
Further insight into miscibility was obtained by means of DMA. The plasticizing effect of $[$ Emim] $[$ TFSI] on the main relaxation ( $\beta$-relaxation) and the broadening of the temperature interval of the relaxation reveals the formation of a homogeneously mixed phase. Figure 2 shows the temperature dependence of the real part of the complex mechanical module $\mathrm{E}^{\prime}$ and $\tan \delta$ for the $\mathrm{PVDF} /[\mathrm{Emim}][\mathrm{TFSI}]$ samples for a fixed frequency $(\mathrm{f}=1 \mathrm{~Hz})$.
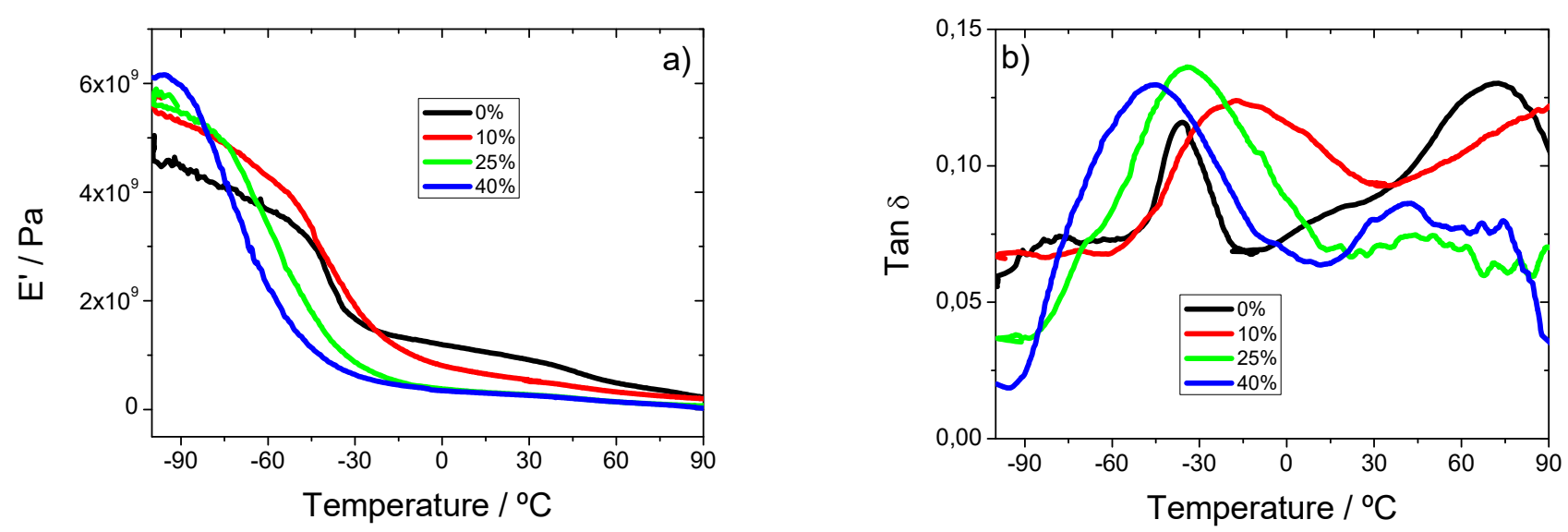

Figure 2. E' (a) and tan $\delta$ (b) vs temperature for the PVDF/[Emim] [TFSI] samples.

The main relaxation process of the PVDF polymer, the $\beta$-relaxation, related with the glass transition, is a broad relaxation process that can be observed in the tan $\delta$ range between 50 and $-10{ }^{\circ} \mathrm{C}$. At higher temperatures (above $60^{\circ} \mathrm{C}$ ) the $\alpha_{c}$-relaxation appears. The latter relaxation is ascribed to molecular motions within the crystalline fraction of the material [45].

Figure 2a) shows the real part of the complex elastic modulus $E^{\prime}$ with the two decreasing steps corresponding to the two abovementioned relaxation processes. Figure $2 b$ ) shows the $\tan \delta$ peak from -90 to $30{ }^{\circ} \mathrm{C}$ corresponding to the $\beta$-relaxation for all the PVDF/[Emim][TFSI] samples. The strength in the E' decrease as the IL content increases. In both diagrams the important broadening of the temperature interval of the $\beta$-relaxation is in perfect agreement with the DSC results. The shift of the relaxation towards lower 
temperatures, the increase of its strength and the broadening are all indications of the participation of the $[$ Emim] $[\mathrm{TFSI}]$ molecules in the cooperative conformational rearrangements of the PVDF chains, and thus the mixing of both components at the molecular scale. Interestingly, the strength of the $\alpha_{c}$-relaxation, associated with the movements of the crystallites, rapidly decreases with the increase of IL content, and in fact the step in E' cannot be observed in the traces of Figure 2a, although a small, broad peak in $\tan \delta$ appears in Figure 2b.

In Figure $2 b$ ) in the case of the PVDF/[Emim][TFSI]10\% sample the onset of the tan $\delta$ peak for $\beta$-relaxation is nearly coincident with that of pure PVDF. Nevertheless, since the relaxation is much broader in the blend, the relaxation peak extends towards higher temperatures and the maximum appears at temperatures slightly higher than in pure PVDF. This result is also in agreement with the DSC curves extended (Figure 2b), in which the peak for $\alpha_{c}$-relaxation is also observed at temperatures slightly higher than pure PVDF. Also the compatibility between this IL and PVDF was observed in [27] by the FTIR technique where the addition of IL in PVDF leads to the nucleation of the beta phase due to the strong electrostatic interaction between the negative charge of the IL anion and the positive side of the PVDF dipole moments.

\subsection{Dielectric analysis}

\subsubsection{Dielectric and electric modulus formalism}

The temperature dependence of the real and imaginary parts of the complex dielectric permittivity for the PVDF/[Emim][TFSI] samples at $1 \mathrm{~Hz}$ is shown in Figure 3 a) and $3 b)$, respectively.

For all PVDF/[Emim][TFSI] samples $\varepsilon^{\prime}$ and $\varepsilon^{\prime \prime}$ increase with increasing temperature due basically to different processes: DC conductivity, electrode polarization, MaxwellWagner relaxation and charge motion and transport [28]. Moreover, it is verified that both 
$\varepsilon^{\prime}$ and $\varepsilon^{\prime \prime}$ increase with increasing IL content due to the decoupling and ion transport of the IL, resulting in higher number of carriers in the polymer matrix [26]. In Figure 3b) only for neat PVDF a peak around $-40{ }^{\circ} \mathrm{C}$, related to the $\beta$-relaxation, is detected. For the other samples, this peak is not observed due to the overlapping of the conductivity component. Figures 3c) and 3d) show the real and imaginary part of the complex dielectric permittivity, respectively, as a function of frequency for PVDF/[Emim][TFSI] samples at $20{ }^{\circ} \mathrm{C}$ where the effect of the electrode polarization is detected. The large values of $\varepsilon^{\prime}$ and $\varepsilon^{\prime \prime}$ at low frequencies for samples with high IL content are due to the electrode polarization effect caused by the accumulation of ionic charge carriers at the electrode-sample interface [46].

The electric modulus formalism provides a more clear characterization of the dipolar relaxation mechanisms.
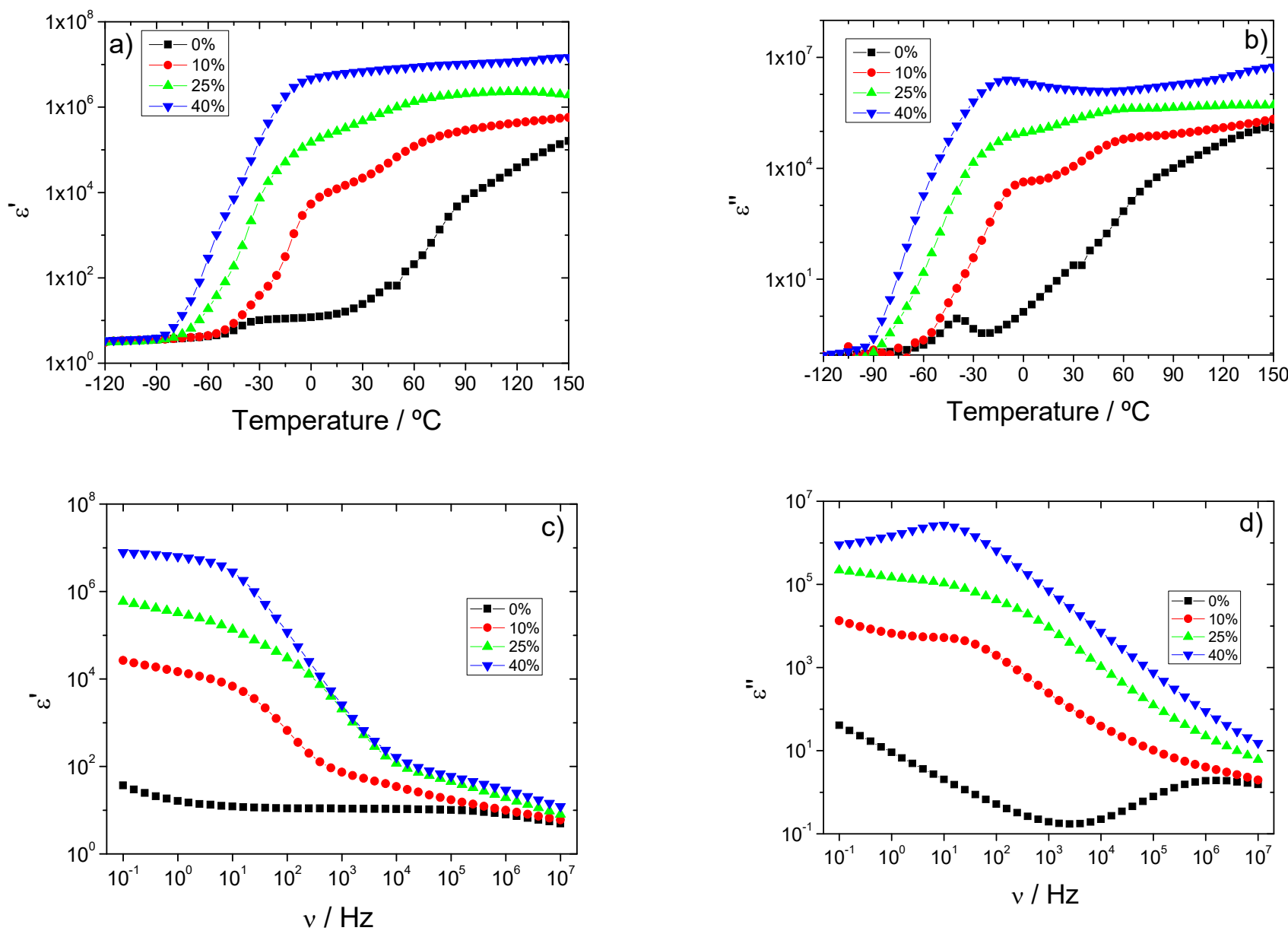
Figure 3. $\varepsilon^{\prime}$ (a and c) and $\varepsilon^{\prime \prime}$ ( $\mathrm{b}$ and d) vs temperature at $1 \mathrm{~Hz}$ and frequency for $20^{\circ} \mathrm{C}$, respectively for all PVDF/IL samples.

The complex electric modulus $\mathrm{M}^{*}$ is defined as the reciprocal of the complex relative permittivity, $\varepsilon^{*}(\omega)$ :

$$
M^{*}(\omega)=1 / \varepsilon^{*}(\omega)=M^{\prime}(\omega)+i M^{\prime \prime}(\omega)=\frac{\varepsilon^{\prime}(\omega)}{\varepsilon^{\prime 2}(\omega)+\varepsilon^{\prime 2}(\omega)}+i \frac{\varepsilon^{\prime \prime}(\omega)}{\varepsilon^{\prime 2}(\omega)+\varepsilon^{\prime 2}(\omega)}
$$

where $\mathrm{M}^{\prime}, \mathrm{M}^{\prime \prime}$ are the real and imaginary components of the complex electric modulus, respectively.

Figure 4 shows the imaginary part of the complex modulus for all PVDF/[Emim][TFSI] samples between -90 and $10{ }^{\circ} \mathrm{C}$, where the $\beta$-relaxation is detected. Another dipolar relaxation, named $\gamma$-relaxation, is detected for neat PVDF and for $\mathrm{IL}=10 \%$ at low temperatures below $-80^{\circ} \mathrm{C}$. The latter relaxation is ascribed to local mobility [45]. It can also be observed that the $\mathrm{M}$ " spectra are asymmetric when the temperature increases and skewed towards for higher frequencies for all the doped samples, suggesting fast ionic motion [47].

The relaxation time for each isochronal plot is calculated from the frequency of the maximum of $\mathrm{M}$ " using the following equation:

$$
\tau=\frac{1}{2 \pi v}
$$

where $\tau$ is the relaxation time and $v$ is the frequency. 

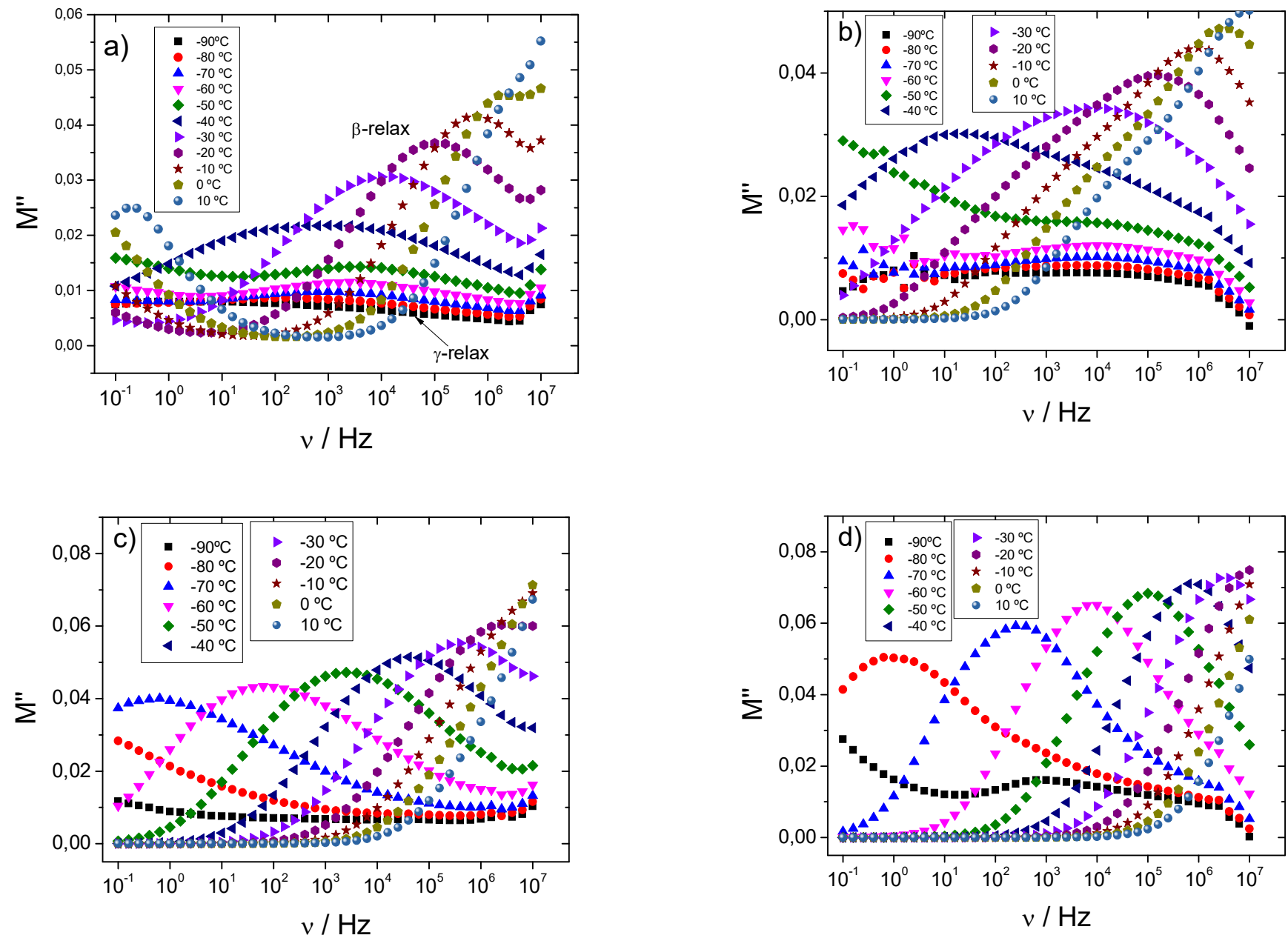

Figure 4. Isothermal spectra of the imaginary part of the complex modulus for neat PVDF

(a), and for $\mathrm{PVDF} /[$ Emim $][\mathrm{TFSI}] 10 \%$ (b), PVDF/[Emim] $][\mathrm{TFSI}] 25 \%$ (c) and $\mathrm{PVDF} /[$ Emim] $][\mathrm{TFSI}] 40 \%$ (d).

The dynamics of the $\beta$-relaxation observed for the PVDF/[Emim][TFSI] samples (Figure

4) was analysed in the scope of the Vogel-Fulcher-Tammann (VFTH) formalism [48]:

$$
\tau(T)=\tau_{0} e^{\frac{B}{T-T_{0}}}
$$


where $\tau_{0}$ is the relaxation time for infinite temperature, $\mathrm{B}$ is a constant and $\mathrm{T}_{0}$ (Vogel temperature) is the temperature where all the chain motions in equilibrium are frozen (usually $30-70 \mathrm{~K}$ below $\mathrm{T}_{\mathrm{g}}$ ).

The fitting of Eq. (4) to the experimental results and the fitting parameters for $\beta$-relaxation are represented in Figure 5 and Table 2, respectively.

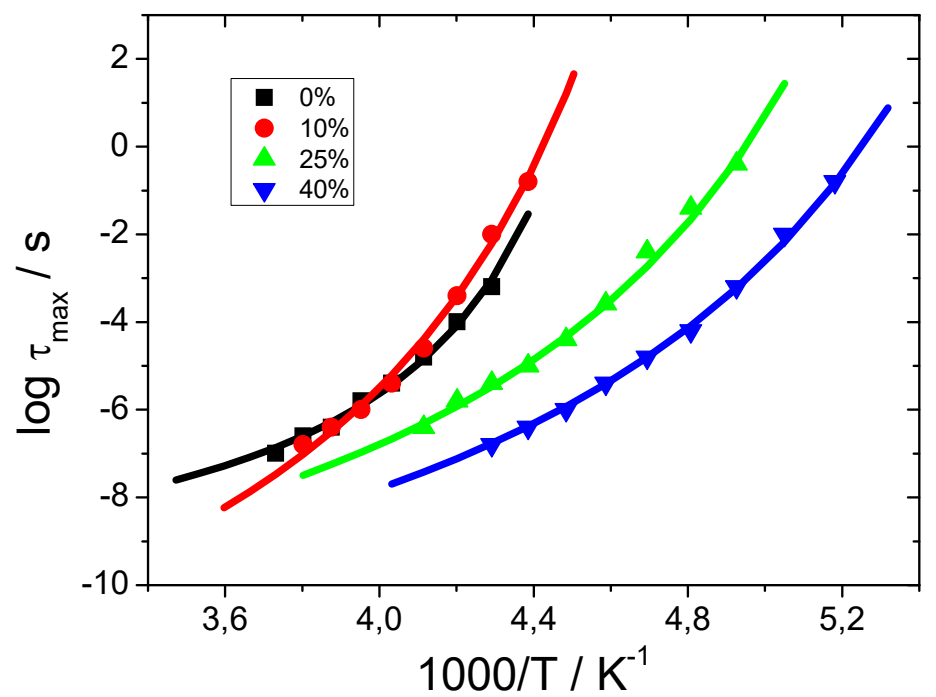

Figure 5. VFTH fitting for the $\beta$-relaxation obtained from M'” (isothermal curves).

If we replace the VFTH expression (Eq. (4)) in the apparent activation energy equation as a function of temperature:

$$
E_{a}(T)=\frac{d \ln \tau}{d \frac{1}{T}}=\frac{R \cdot B}{\left(1-T_{0} / T\right)^{2}}
$$

where $\mathrm{R}$ is ideal gas constant.

From the VTFH fitting parameters, the fragility parameter [49] is calculated:

$$
m\left(T_{g}\right)=\frac{E_{a}\left(T_{g}\right)}{\ln 10 R \cdot T_{g}}
$$


where $m$ is an indication of the steepness of the variation of the material properties (viscosity, relaxation time, ...) as $T_{g}$ is reached. A high $m$ value defines a fragile material, whereas a strong material is characterized by small $m$ values [50].

We have estimated the dielectric glass transition temperature, Tg-diel, by replacing $\tau$ by $230 \mathrm{~s}$ in Eq. (4). Table 2 shows all the VFTH fitting parameters and the activation energy of the $\beta$-relaxation for the PVDF/[Emim][TFSI] samples.

Table 2. VFTH fitting parameter and apparent activation energy ( $\tau=230 \mathrm{~s})$ obtained for the $\beta$-relaxation of the PVDF/[Emim][TFSI] samples.

\begin{tabular}{cccccccc}
\hline & $\begin{array}{c}\text { Temperature } \\
\text { range } /{ }^{\circ} \mathrm{C}\end{array}$ & $\tau_{0}(\mathrm{~s})$ & $\mathrm{B}(\mathrm{K})$ & $\mathrm{T}_{0}(\mathrm{~K})$ & $\begin{array}{c}\mathrm{E}_{\mathrm{a}}\left(\mathrm{KJmol}^{-1}\right) \\
\tau=230 \mathrm{~s}\end{array}$ & $\begin{array}{c}\mathrm{T}_{\mathrm{g}-} \\
\text { diele } /\end{array}$ & $\mathrm{m}$ \\
Samples & & & & & & \\
\hline PVDF & {$[-45,-5]$} & $1.18 \mathrm{E}-10$ & 445 & $2.05 \mathrm{E}+02$ & 729 & -52 & 179 \\
$10 \%$ & {$[-50,-5]$} & $4.73 \mathrm{E}-15$ & 1270 & $1.87 \mathrm{E}+02$ & 470 & -53 & 111 \\
$25 \%$ & {$[-70,-20]$} & $7.01 \mathrm{E}-13$ & 1160.7 & $1.64 \mathrm{E}+02$ & 321 & -76 & 86 \\
$40 \%$ & {$[-80,-35]$} & $2.70 \mathrm{E}-13$ & 1057.2 & $1.54 \mathrm{E}+02$ & 316 & -88 & 89 \\
\hline
\end{tabular}

The main dielectric relaxation in an amorphous polymer is due to chain segment rearrangements involving a region of the material called cooperative rearranging region, CRR with a characteristic dimension that increases with decreasing temperature, reaching a value of few nanometers at $T_{g}$ [51-54]. The presence of [Emim][TFSI] inside the CRR mixed with the PVDF chain segments decreased the total energy involved in the rearrangement because individual ions of the IL are more mobile than the chain segments due to chain connectivity. Further, figure 5 also shows a crossing of the VFTH characteristic curves for neat PVDF and PVDF/[Emim][TFSI $] 10 \%$ due to the increase of the Maxwell-Wagner effect and dc conductivity induced by the presence of the IL.

This is why the $\mathrm{E}_{\mathrm{a}}$ decreases with increasing IL content in the blend and the curvature of the $\ln \tau$ vs $1 /$ T curves of Figure 5 decreases (the material becomes stronger, fragility decreases) while shifting towards lower temperatures when the fraction of [Emim][TFSI] in the blend is higher. 
The $\tau_{0}$ factor for samples with IL amount is of the order $>10^{-13} \mathrm{~s}$, as the typical values for non-cooperative Debye process originated by local orientational fluctuations [55].

A rough estimate of the equivalent frequency of DSC can be obtained within Donth's fluctuation model [56]:

$$
f_{e q, D S C}=\frac{q^{\prime}}{2 \pi \alpha \delta T}
$$

where $q^{\prime}$ is the cooling rate, $\alpha$ is a constant on the order of 1 , and $\delta T$ is the mean temperature fluctuation (on the order of $2{ }^{\circ} \mathrm{C}$ ). In this work, the $\mathrm{f}_{\mathrm{eq}, \mathrm{DSC}}$ is $0.027 \mathrm{~Hz}$, or a relaxation time of $\tau_{T g}=230 \mathrm{~s}$. The $\mathrm{T}_{\mathrm{g}}$ values, determined as the temperature at which the dielectric relaxation time is $\tau_{T g}$, are listed in Table 2, showing the good agreement between BDS and calorimetric data for all the PVDF/[Emim][TFSI] samples.

\subsubsection{Conductivity formalism}

To understand the relaxation dynamics of the transport of charge carriers and the electrode polarization, it is appropriate to represent the dependence of frequency and temperature of the complex conductivity, $\sigma^{*}$, which can be calculated from the dielectric function by the following equation:

$$
\sigma^{*}=\sigma^{\prime}(\omega)+i \sigma^{\prime \prime}(\omega)
$$

Thus, the real part of the conductivity is given by:

$$
\sigma^{\prime}(\omega)=\varepsilon_{0} \omega \varepsilon^{\prime \prime}(\omega)
$$

and its imaginary part is given by:

$$
\sigma^{\prime \prime}(\omega)=\varepsilon_{0} \omega \varepsilon^{\prime}(\omega)
$$

where $\varepsilon_{0}\left(8.85 \times 10^{-12} \mathrm{Fm}^{-1}\right)$ is the permittivity of free space and $\omega=2 \pi v$ is the angular frequency. 
The real and imaginary parts of the $\sigma^{*}(\omega)$ are shown in Figure 6 for neat PVDF and for $\mathrm{X}=40 \%$. The conductivity behaviour of the other samples is between that of these samples. For neat PVDF (Figures 6a and 6b), $\sigma^{\prime}$ and $\sigma^{\prime \prime}$ increase with increasing frequency for all temperatures. This is related to hopping transport of localized charge carriers and is described by the following equation [57]:

$$
\sigma^{\prime}(\omega) \propto \omega^{n}, n \leq 1
$$

For the whole temperature range, the exponent $n$ is the $0.13<n<0.9$ range for neat PVDF.

For PVDF/[Emim][TFSI $] 10 \%$ at lower temperatures (below $10^{\circ} \mathrm{C}$ ) (see Supplementary Information, figure S2), frequency dependence of the conductivity follows Eq. (11) with $n \sim 0.7$ a behaviour reported for charge carrier hopping. For this sample, but at temperatures above $20^{\circ} \mathrm{C}$, a plateau of $\sigma^{\prime}$, that represents the DC conductivity, followed by an increase of $\sigma$ ' with frequency, due to electrode polarisation, are evident [34]. The plateau is more pronounced for PVDF/[Emim][TFSI] with IL $>10 \%$ (Figure $6 \mathrm{c}$ )). On the other hand, the frequency of the maximum in $\sigma^{\prime \prime}\left(f_{\max }\right)$ shown in Figure $6 \mathrm{~d}$, which corresponds to the full development of electrode polarization, is better separated from the position $f_{\text {on }}$ of the local minimum of $\sigma^{\prime \prime}$, indicating the onset of electrode polarization, as indicated in Figure 6d) [34]. The position and intensity of $f_{\text {on }}$ and $f_{\max }$ increase with the increase of IL content. 

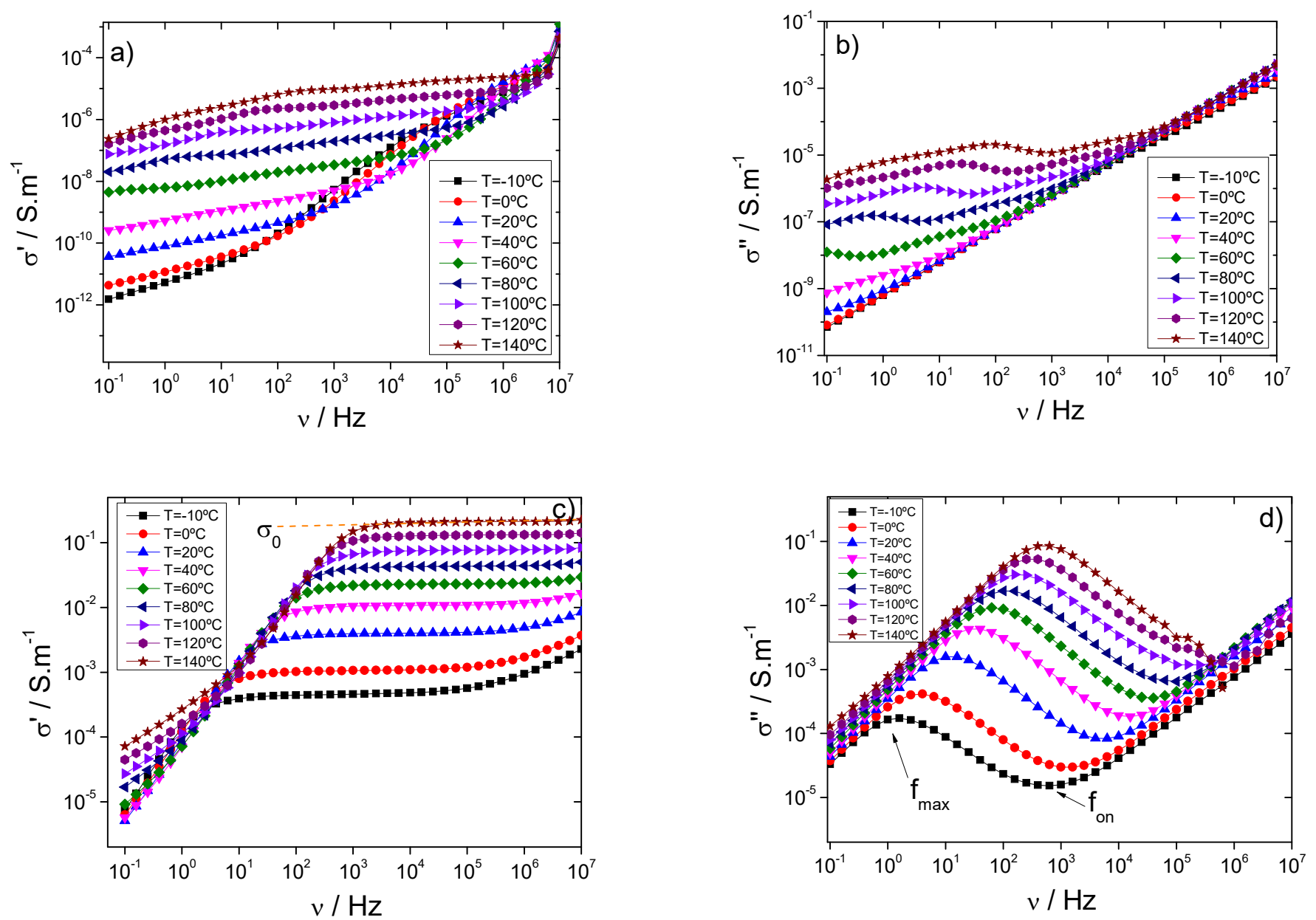

Figure 6. Frequency dependence of the real and imaginary part of the conductivity $\left(\sigma^{*}\right)$ at different temperatures for neat PVDF ( $a$ and $b$ ) and PVDF/[Emim][TFSI] 40\% (c and d).

Considering that charge transport mechanism at the metal-ion interfaces is described by electrode polarization, Serghei proposed a theoretical approach to calculate the DC conductivity by the following equation into ILs [34]:

$$
\sigma_{D C}=2 \pi \varepsilon_{0} \varepsilon_{s} \frac{f_{o n}^{2}}{f_{\max }}
$$

where $\varepsilon_{0}$ is the permittivity of free space and $\varepsilon_{S}$ is the static permittivity of the bulk material measured at $\mathrm{f}>\mathrm{f}_{\text {on }}$. 
The DC conductivity obtained from the $\sigma$, plateau, illustrated in Figure 6c) for $\mathrm{PVDF} /[$ Emim] $][\mathrm{TFSI}] 40 \%$, is shown in Figure $7 \mathrm{a}$ ) for all the PVDF/[Emim][TFSI] samples at different temperatures.
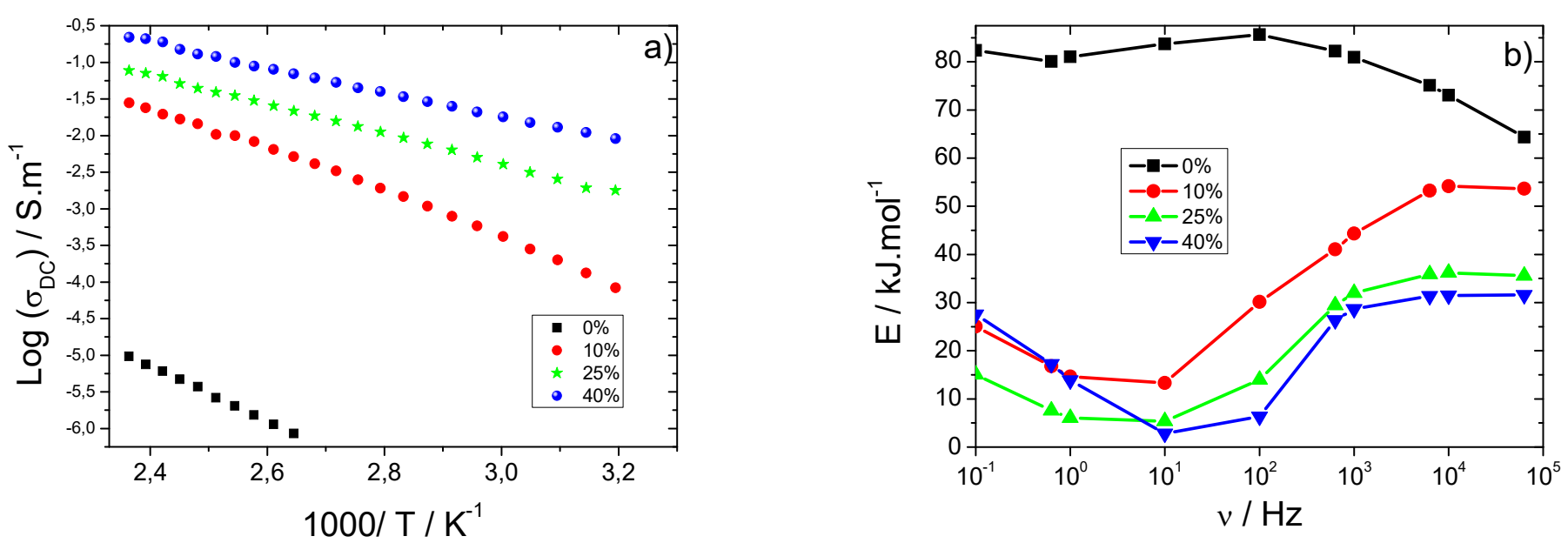

Figure 7. DC conductivity as a function of temperature a) and evolution of the apparent activation energy obtained after fittings with the Dyre model b) for the PVDF/[Emim][TFSI] samples.

As shown in Figure 7a), DC conductivity increases with the increase of [Emim][TFSI] content because the dissociation and ion transport of the IL results in higher number of charge carriers in the polymer matrix [58].

For the evaluation of the dependence of the AC conductivity with temperature at different frequencies, the Dyre model (random free energy barrier model or symmetric model) was applied [59]:

$$
\sigma(T)=B e^{\frac{E}{R \cdot N_{A}^{-1} \cdot T}}
$$

where $B$ is a pre-exponential factor identified as the attempt frequency, $E$ is the apparent activation energy of the process, $\mathrm{T}$ is temperature, $\mathrm{R}$ is ideal gas constant and $N_{A}$ is the Avogadro's number. 
The application of the Dyre model was performed considering the real part of the conductivity $\left(\sigma^{\prime}\right)$ between $40{ }^{\circ} \mathrm{C}$ (above $T_{g}$ ) and $140{ }^{\circ} \mathrm{C}$ and in the frequency range between $0.1 \mathrm{~Hz}$ and $65 \mathrm{kHz}$. The activation energy, $E$, values calculated are plotted in Figure $7 \mathrm{~b}$ ) as a function of frequency.

In the PVDF sample, the E value decreases with increasing frequency above $10^{3} \mathrm{~Hz}$ (the change from dc to ac conduction for temperatures above $40^{\circ} \mathrm{C}$ in Fig. 6)). Figure $7 \mathrm{~b}$ ) shows that the addition of the IL leads to the sharp reduction of the activation energy of the process for all frequencies with respect to PVDF, as well as frequency dependence changes. Above $1 \mathrm{~Hz}, \mathrm{E}$ decreases with the increasing incorporation of [Emim][TFSI] into PVDF matrix. For PVDF/[Emim][TFSI] $40 \%$ at lower frequencies up to $10^{1} \mathrm{~Hz}$, the activation energy decreases very rapidly due to the decoupling of the translation movements and the high number of charge carriers.

Between 0.1 and $1 \mathrm{~Hz}$, the $\mathrm{E}$ value decreases with increasing frequency due to the electrode polarization effect into PVDF/[Emim][TFSI] samples. Above of $10^{4} \mathrm{~Hz}$ for $\mathrm{IL}=10 \%$ and $10^{3}$ for $\mathrm{IL}=25 \%$ and $40 \%$, the $\mathrm{E}$ value remains practically constant (Figure 7b). The reason for this behavior is correlated to the plateau zone of Figure 6. 


\section{Conclusions}

The thermal, mechanical and dielectric properties of PVDF/[Emim][TFSI] blends with different IL contents ( $10 \% \leq \mathrm{IL} \leq 40 \%)$ were characterized to determine the influence of [Emim][TFSI] on the polymer's segmental dynamics and on ionic conductivity. All the techniques showed, not only that IL and PVDF are miscible, but also that the chain segments and the IL are mixed at the nanometer range. This was demonstrated by the shift of the glass transition or the main dielectric or dynamic-mechanical main relaxation towards lower temperatures. Furthermore, the broadening due to composition fluctuations and the fragility of the material decreased due to the participation of the IL in the cooperative rearrangements of the PVDF chain segments. For all the $\mathrm{PVDF} /[\mathrm{Emim}][\mathrm{TFSI}]$ samples, a good agreement between the $\mathrm{T}_{\mathrm{g}}$ determined by BDS and DSC was found.

The conductivity formalism revealed that the IL affected the conductivity behavior of the blends from the standpoint of charge motion, ion concentration and electrode polarization effects. For all the doped samples, DC conductivity increased and the apparent activation energy decreased with the increase of IL content. The high DC conductivity value of the [Emim][TFSI]-doped samples in comparison to neat PVDF is due to the ionic dissociation and ion transport of the IL resulting in a higher carrier numbers in the polymer matrix. 


\section{Acknowledgements}

This work was supported by the Portuguese Foundation for Science and Technology (FCT) in the framework of the Strategic Funding UID/FIS/04650/2013. The authors thank FEDER funds through the COMPETE 2020 Programme and National Funds through FCT under the projects PTDC/CTM-ENE/5387/2014, PTDC/EEI-SII/5582/2014 and $\mathrm{UID} / \mathrm{CTM} / 50025 / 2013$ and Grants SFRH/BPD/121526/2016 (D.M.C.), SFRH/BPD/112547/2015 (C.M.C.). Financial support from the Spanish Ministry of Economy and Competitiveness (MINECO) through the project MAT2016-76039-C4-3R (AEI/FEDER, UE) (including the FEDER financial support) and from the Basque Government Industry Department under the ELKARTEK and HAZITEK programs is also acknowledged. CIBER-BBN is an initiative funded by the VI National R\&D\&I Plan 2008-2011, Iniciativa Ingenio 2010, Consolider Program. CIBER Actions are financed by the Instituto de Salud Carlos III with assistance from the European Regional Development Fund. 


\section{References}

[1] M.M. Thackeray, C. Wolverton, E.D. Isaacs, Electrical energy storage for transportation-approaching the limits of, and going beyond, lithium-ion batteries, Energy \& Environmental Science 5(7) (2012) 7854-7863.

[2] G. Majeau-Bettez, T.R. Hawkins, A.H. Strømman, Life Cycle Environmental Assessment of Lithium-Ion and Nickel Metal Hydride Batteries for Plug-In Hybrid and Battery Electric Vehicles, Environmental Science \& Technology 45(10) (2011) 45484554.

[3] B. Nykvist, M. Nilsson, Rapidly falling costs of battery packs for electric vehicles, Nature Clim. Change 5(4) (2015) 329-332.

[4] A. Arya, A.L. Sharma, Polymer electrolytes for lithium ion batteries: a critical study, Ionics 23(3) (2017) 497-540.

[5] P.V. Wright, Polymer electrolytes - the early days, Electrochimica Acta 43(10-11) (1998) 1137-1143.

[6] F.M. Gray, R.S.o. Chemistry, Polymer electrolytes, Royal Society of Chemistry1997.

[7] M. Popall, M. Andrei, J. Kappel, J. Kron, K. Olma, B. Olsowski, ORMOCERs as inorganic-organic electrolytes for new solid state lithium batteries and supercapacitors, Electrochimica Acta 43(10-11) (1998) 1155-1161.

[8] V. Di Noto, S. Lavina, G.A. Giffin, E. Negro, B. Scrosati, Polymer electrolytes: Present, past and future, Electrochimica Acta 57(0) (2011) 4-13.

[9] K. Murata, An overview of the research and development of solid polymer electrolyte batteries, Electrochimica Acta 40(13-14) (1995) 2177-2184.

[10] C.M. Costa, M.M. Silva, S. Lanceros-Mendez, Battery separators based on vinylidene fluoride (VDF) polymers and copolymers for lithium ion battery applications, RSC Advances 3(29) (2013) 11404-11417.

[11] H.S. Nalwa, Ferroelectric Polymers: Chemistry, Physics, and Applications, M. Dekker, Incorporated 1995.

[12] L. Rasmussen, Electroactivity in Polymeric Materials, Springer2012.

[13] X. Xue, S. Wang, W. Guo, Y. Zhang, Z.L. Wang, Hybridizing Energy Conversion and Storage in a Mechanical-to-Electrochemical Process for Self-Charging Power Cell, Nano Letters 12(9) (2012) 5048-5054.

[14] Z. Wang, Modeling and Simulation of Piezoelectrically Driven Self-Charging Lithium Ion Batteries, ACS Applied Materials \& Interfaces 9(18) (2017) 15893-15897. 
[15] Y.-S. Ye, J. Rick, B.-J. Hwang, Ionic liquid polymer electrolytes, Journal of Materials Chemistry A 1(8) (2013) 2719-2743.

[16] M.J. Park, I. Choi, J. Hong, O. Kim, Polymer electrolytes integrated with ionic liquids for future electrochemical devices, Journal of Applied Polymer Science 129(5) (2013) 2363-2376.

[17] K.N. Marsh, J.A. Boxall, R. Lichtenthaler, Room temperature ionic liquids and their mixtures - a review, Fluid Phase Equilibria 219(1) (2004) 93-98.

[18] H. Niedermeyer, J.P. Hallett, I.J. Villar-Garcia, P.A. Hunt, T. Welton, Mixtures of ionic liquids, Chemical Society Reviews 41(23) (2012) 7780-7802.

[19] A. Lewandowski, A. Świderska-Mocek, Ionic liquids as electrolytes for Li-ion batteries-An overview of electrochemical studies, Journal of Power Sources 194(2) (2009) 601-609.

[20] C. Xing, Y. Wang, C. Zhang, L. Li, Y. Li, J. Li, Immobilization of Ionic Liquids onto the Poly(vinylidene fluoride) by Electron Beam Irradiation, Industrial \& Engineering Chemistry Research 54(38) (2015) 9351-9359.

[21] C. Xing, J. You, Y. Li, J. Li, Nanostructured Poly(vinylidene fluoride)/Ionic Liquid Composites: Formation of Organic Conductive Nanodomains in Polymer Matrix, The Journal of Physical Chemistry C 119(36) (2015) 21155-21164.

[22] J. Guan, C. Xing, Y. Wang, Y. Li, J. Li, Poly (vinylidene fluoride) dielectric composites with both ionic nanoclusters and well dispersed graphene oxide, Composites Science and Technology 138 (2017) 98-105.

[23] C. Xing, J. Li, C. Yang, Y. Li, Local Grafting of Ionic Liquid in Poly(vinylidene fluoride) Amorphous Region and the Subsequent Microphase Separation Behavior in Melt, Macromolecular Rapid Communications 37(19) (2016) 1559-1565.

[24] C. Xing, Y. Wang, X. Huang, Y. Li, J. Li, Poly(vinylidene fluoride) Nanocomposites with Simultaneous Organic Nanodomains and Inorganic Nanoparticles, Macromolecules 49(3) (2016) 1026-1035.

[25] C. Xing, M. Zhao, L. Zhao, J. You, X. Cao, Y. Li, Ionic liquid modified poly(vinylidene fluoride): crystalline structures, miscibility, and physical properties, Polymer Chemistry 4(24) (2013) 5726-5734.

[26] J.C. Dias, M.S. Martins, S. Ribeiro, M.M. Silva, J.M.S.S. Esperança, C. Ribeiro, G. Botelho, C.M. Costa, S. Lanceros-Mendez, Electromechanical actuators based on poly(vinylidene fluoride) with [N1 $1112(\mathrm{OH})][\mathrm{NTf} 2]$ and [C2mim] [C2SO4], Journal of Materials Science 51(20) (2016) 9490-9503. 
[27] J.C. Dias, A.C. Lopes, B. Magalhães, G. Botelho, M.M. Silva, J.M.S.S. Esperança, S. Lanceros-Mendez, High performance electromechanical actuators based on ionic liquid/poly(vinylidene fluoride), Polymer Testing 48 (2015) 199-205.

[28] J.R. Sangoro, F. Kremer, Charge Transport and Glassy Dynamics in Ionic Liquids, Accounts of Chemical Research 45(4) (2012) 525-532.

[29] Y. Jiang, H. Nadolny, S. Kashammer, S. Weibels, W. Schroer, H. Weingartner, The ion speciation of ionic liquids in molecular solvents of low and medium polarity, Faraday Discussions 154(0) (2012) 391-407.

[30] S. Agrawal, M. Singh, M. Tripathi, M. Dwivedi, K. Pandey, Dielectric relaxation studies on [PEO-SiO 2$]: \mathrm{NH}_{4} \mathrm{SCN}$ nanocomposite polymer electrolyte films, Journal of Materials Science 44(22) (2009) 6060-6068.

[31] S. Havriliak, S.J. Havriliak, Dielectric and Mechanical Relaxation in Materials: Analysis, Interpretation, and Application to Polymers, Hanser Publishers 1997.

[32] F. Kremer, A. Schönhals, Broadband Dielectric Spectroscopy, Springer2003.

[33] K. Nakamura, T. Saiwaki, K. Fukao, Dielectric Relaxation Behavior of Polymerized Ionic Liquid, Macromolecules 43(14) (2010) 6092-6098.

[34] A. Serghei, M. Tress, J.R. Sangoro, F. Kremer, Electrode polarization and charge transport at solid interfaces, Physical Review B 80(18) (2009) 184301.

[35] D. Fragiadakis, S. Dou, R.H. Colby, J. Runt, Molecular Mobility, Ion Mobility, and Mobile Ion Concentration in Poly(ethylene oxide)-Based Polyurethane Ionomers, Macromolecules 41(15) (2008) 5723-5728.

[36] P. Frübing, F. Wang, T.-F. Kühle, R. Gerhard, AC and DC conductivity of ionic liquid containing polyvinylidene fluoride thin films, Applied Physics A 122(1) (2016) 79. [37] P. Xu, W. Fu, X. Luo, Y. Ding, Enhanced dc conductivity and conductivity relaxation in PVDF/ionic liquid composites, Materials Letters 206 (2017) 60-63.

[38] H. Matsumoto, Recent Advances in Ionic Liquids for Lithium Secondary Batteries, in: T.R. Jow, K. Xu, O. Borodin, M. Ue (Eds.), Electrolytes for Lithium and Lithium-Ion Batteries, Springer New York, New York, NY, 2014, pp. 209-225.

[39] C.M. Costa, H.M. Rodrigues, A. Gören, A.V. Machado, M.M. Silva, S. LancerosMéndez, Preparation of Poly(vinylidene fluoride) Lithium-Ion Battery Separators and Their Compatibilization with Ionic Liquid - A Green Solvent Approach, ChemistrySelect 2(19) (2017) 5394-5402. 
[40] B.-E. El Mohajir, N. Heymans, Changes in structural and mechanical behaviour of PVDF with processing and thermomechanical treatments. 1. Change in structure, Polymer 42(13) (2001) 5661-5667.

[41] Y.U. Paulechka, A.V. Blokhin, G.J. Kabo, A.A. Strechan, Thermodynamic properties and polymorphism of 1-alkyl-3-methylimidazolium bis(triflamides), The Journal of Chemical Thermodynamics 39(6) (2007) 866-877.

[42] M.P. Silva, C.M. Costa, V. Sencadas, A.J. Paleo, S. Lanceros-Méndez, Degradation of the dielectric and piezoelectric response of $\beta$-poly(vinylidene fluoride) after temperature annealing, Journal of Polymer Research 18(6) (2011) 1451-1457.

[43] R. Mejri, J.C. Dias, S.B. Hentati, M.S. Martins, C.M. Costa, S. Lanceros-Mendez, Effect of anion type in the performance of ionic liquid/poly(vinylidene fluoride) electromechanical actuators, Journal of Non-Crystalline Solids 453 (2016) 8-15.

[44] R. Leones, C.M. Costa, A.V. Machado, J.M.S.S. Esperança, M.M. Silva, S. Lanceros-Méndez, Effect of Ionic Liquid Anion Type in the Performance of Solid Polymer Electrolytes Based on Poly(Vinylidene fluoride-trifluoroethylene), Electroanalysis 27(2) (2015) 457-464.

[45] V. Sencadas, S. Lanceros-Méndez, R. Sabater i Serra, A. Andrio Balado, J.L. Gómez Ribelles, Relaxation dynamics of poly(vinylidene fluoride) studied by dynamical mechanical measurements and dielectric spectroscopy, The European Physical Journal E 35(5) (2012) 41.

[46] F. Frenzel, R. Guterman, A.M. Anton, J. Yuan, F. Kremer, Molecular Dynamics and Charge Transport in Highly Conductive Polymeric Ionic Liquids, Macromolecules 50(10) (2017) 4022-4029.

[47] T. Dam, S.S. Jena, D.K. Pradhan, The ionic transport mechanism and coupling between the ion conduction and segmental relaxation processes of PEO20-LiCF3SO3 based ion conducting polymer clay composites, Physical Chemistry Chemical Physics 18(29) (2016) 19955-19965.

[48] R. Sabater i Serra, J.L. Escobar Ivirico, F. Romero Colomer, A. Andrio Balado, J.L. Gómez Ribelles, Molecular dynamics in polymer networks containing caprolactone and ethylene glycol moieties studied by dielectric relaxation spectroscopy, Journal of NonCrystalline Solids 404 (2014) 109-115.

[49] R. Tao, S.L. Simon, Rheology of Imidazolium-Based Ionic Liquids with Aromatic Functionality, The Journal of Physical Chemistry B 119(35) (2015) 11953-11959. 
[50] S. Firmino Mendes, C.M. Costa, V. Sencadas, J. Serrado Nunes, P. Costa, R. Gregorio, S. Lanceros-Méndez, Effect of the ceramic grain size and concentration on the dynamical mechanical and dielectric behavior of poly(vinilidene fluoride)/Pb(Zr0.53Ti0.47)O3 composites, Applied Physics A 96(4) (2009) 899-908.

[51] G. Adam, J.H. Gibbs, On the Temperature Dependence of Cooperative Relaxation Properties in Glass-Forming Liquids, The Journal of Chemical Physics 43(1) (1965) 139146.

[52] E. Donth, Characteristic length of the glass transition, Journal of Polymer Science Part B: Polymer Physics 34(17) (1996) 2881-2892.

[53] J.L. Gómez Ribelles, A. Vidaurre Garayo, J.M.G. Cowie, R. Ferguson, S. Harris, I.J. McEwen, The length of cooperativity at the glass transition in poly(vinyl acetate) from the modeling of the structural relaxation process, Polymer 40(1) (1999) 183-192.

[54] E. Donth, The size of cooperatively rearranging regions at the glass transition, Journal of Non-Crystalline Solids 53(3) (1982) 325-330.

[55] M.T. Viciosa, G. Santos, A. Costa, F. Danede, L.C. Branco, N. Jordao, N.T. Correia, M. Dionisio, Dipolar motions and ionic conduction in an ibuprofen derived ionic liquid, Physical Chemistry Chemical Physics 17(37) (2015) 24108-24120.

[56] A. Vassilikou-Dova, I.M. Kalogeras, Dielectric Analysis (DEA), Thermal Analysis of Polymers, John Wiley \& Sons, Inc.2008, pp. 497-613.

[57] A.K. Jonscher, The /'universal/' dielectric response, Nature 267(5613) (1977) 673679.

[58] R. Leones, C.M. Costa, A.V. Machado, J.M.S.S. Esperança, M.M. Silva, S. Lanceros-Méndez, Development of solid polymer electrolytes based on poly(vinylidene

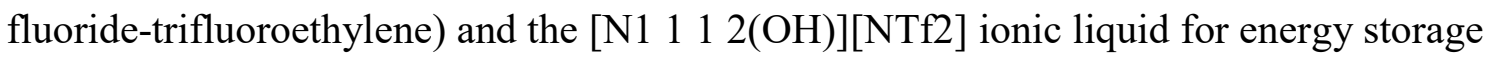
applications, Solid State Ionics 253 (2013) 143-150.

[59] J.C. Dyre, The random free-energy barrier model for ac conduction in disordered solids, Journal of Applied Physics 64(5) (1988) 2456-2468. 